\title{
Walsh equiconvergence theorems in the quaternionic setting
}

\author{
Sorin G. Gal ${ }^{\mathrm{a}}$ and Irene Sabadini ${ }^{\mathrm{b} *}$ \\ ${ }^{a}$ Department of Mathematics and Computer Science, University of Oradea, Str. Universitatii Nr. 1, \\ Oradea 410087, Romania, ${ }^{b}$ Dipartimento di Matematica, Politecnico di Milano, \\ Via Bonardi 9, Milano 20133, Italy \\ Communicated by $\mathrm{H}$. Begehr
}

(Received 10 April 2013; accepted 18 November 2013)

\begin{abstract}
In this paper, we prove the quaternionic version of the result of Walsh stating that the difference between the partial sums of the Taylor expansion of an analytic function and its interpolation polynomial at the roots of unity converges in a larger disc than the disc of analyticity of the function. Our result holds for functions of a quaternionic variable which are slice regular in a ball and thus they admit a converging power series expansion. We also prove a generalization of this theorem as well as its converse. Because of the noncommutative setting, the results are nontrivial and require a notion of multiplication of functions (and of polynomials) which does not commute with the evaluation.
\end{abstract}

Keywords: quaternionic polynomials; slice regular functions; interpolating polynomials

AMS Subject Classifications: 30G35; 12E15; 41A10

\section{Introduction}

For $\rho>1$ and $\mathbb{D}_{\rho}=\{z \in \mathbb{C} ;|z|<\rho\}$, let us denote $A_{\rho}(\mathbb{C})=\left\{f: \mathbb{D}_{\rho} \rightarrow \mathbb{C} ; f\right.$ is analytic in $\left.\mathbb{D}_{\rho}\right\}$. Since $f \in A_{\rho}(\mathbb{C})$ we can write $f(z)=\sum_{k=0}^{\infty} z^{k} a_{k}$ (with $a_{k} \in \mathbb{C}$ ), and the partial $\operatorname{sum} s_{n-1}(f)(z)=\sum_{k=0}^{n-1} z^{k} a_{k}$.

Let $L_{n-1}(f)(z)$ be the Lagrange interpolation polynomial of degree $\leq n-1$, interpolating $f$ on the $n$ zeros of the equation $z^{n}-1=0$. It was known that both $s_{n-1}(f)(z)$ and $L_{n-1}(f)(z)$ converge uniformly in any closed disc of radius $R<\rho$, however Walsh proved the surprising result that the difference $s_{n-1}(f)(z)-L_{n-1}(f)(z)$ converges uniformly to zero in a larger set, namely in any closed disc in $\mathbb{D}_{\rho^{2}}$. This phenomenon is called Walsh equiconvergence and can be stated as follows.

Theorem 1.1 (see e.g. [1], Chapter 1, p.8, Theorem 6, or Walsh [2], p.153-154) If $f \in A_{\rho}(\mathbb{C})$, then $L_{n-1}(f)(z)$ converges geometrically to $f(z)$ in any closed disc $\overline{\mathbb{D}_{R}}$ with $1<R<\rho$ and, in addition

$$
\lim _{n \rightarrow \infty}\left[s_{n-1}(f)(z)-L_{n-1}(f)(z)\right]=0, \quad \text { for all }|z|<\rho^{2},
$$

*Corresponding author. Email: irene.sabadini@polimi.it 
the convergence being again uniform and with geometric rate of convergence in any $\overline{\mathbb{D}_{R}}$ with $1<R<\rho^{2}$. The result is the best possible, in the sense that for the function $g \in A_{\rho}(\mathbb{C})$ given by $g(z)=\frac{1}{z-\rho}$, for $z=\rho^{2}$, we have $\lim _{n \rightarrow \infty}\left[s_{n-1}(g)(z)-L_{n-1}(g)(z)\right] \neq 0$.

In this paper, we address the problem of proving the generalization of Walsh equiconvergence result in the quaternionic framework. This problem is particularly interesting for several reasons. First of all, polynomials with quaternionic coefficients may be written with coefficients on the left, on the right or both sides. In the literature, see e.g. [3], all these situations are considered, however the majority of the results have been obtained in the case in which the polynomials are unilateral, i.e. with coefficients on one side, see [4]. In this paper, we will consider the case of coefficients written on the right (the other case is similar) since they can be seen as a special case of (left) slice regular functions for which there are integral representation like the Cauchy integral formula. Thus, from now on, by the word polynomial we will mean a polynomial of the form $p(q)=q^{n} a_{n}+\cdots+q a_{1}+a_{0}, a_{k} \in \mathbb{H}$. Two such polynomials can be multiplied as in [4]: given $p_{1}(q)=q^{n} a_{n}+\cdots+q a_{1}+a_{0}$, $p_{2}(q)=q^{m} b_{m}+\cdots+q b_{1}+b_{0}$, we define their multiplication that we denote by $*$

$$
\left(p_{1} * p_{2}\right)(q)=\sum_{k=0}^{m+n} q^{k} c_{k}, \quad c_{k}=\sum_{i+j=k} a_{i} b_{j} .
$$

Given a polynomial $p(q)$ as above, its evaluation at the point $q=\alpha$ is defined in the standard way as $p(\alpha)=\sum_{k=0}^{n} \alpha^{k} a_{k}$. The notion of evaluation does not work well with respect to the $*$-multiplication, in fact is not a ring homomorphism: if $r(q)=\left(p_{1} * p_{2}\right)(q)$, then in general $r(\alpha) \neq p(\alpha) q(\alpha)$. To convince themselves, it is sufficient to consider the following simple example: let $r(q)=(q-i) *(q-j)=q^{2}-q(i+j)+k$ then $r(j) \neq 0$ but $(j-i)(j-j)=0$. For this reason, the standard procedure to construct interpolating polynomials does not work in the quaternionic setting. Indeed, in the complex case, given the distinct points $z_{1}, \ldots, z_{n}$ and the values $w_{1}, \ldots, w_{n}$, the polynomial interpolating these values can be constructed according to the so-called Lagrange interpolation formula (see e.g. [5], p.50):

$$
p(z)=\sum_{k=1}^{n} w_{k} \frac{w(z)}{\left(z-z_{k}\right) w^{\prime}(z)}
$$

where $w(z)=\left(z-z_{1}\right) \cdots\left(z-z_{n}\right)$. This formula is based on the fact that $w(z) /\left(z-z_{k}\right)$ vanishes at all points $z_{i}$ different from $z_{k}$. In the quaternionic framework, this procedure cannot work and we will show this fact with a simple example.

Example 1 Consider two distinct points $q_{1}$ and $q_{2}$, and the polynomial $w(q)=$ $\left(q-q_{1}\right) *\left(q-q_{2}\right)$. This polynomial vanishes at $q_{1}$ and at $\tilde{q}_{2}=\left(q_{2}-q_{1}\right)^{-1} q_{2}\left(q_{2}-q_{1}\right)$ so it interpolates the points $\left(q_{1}, 0\right)$ and $\left(\tilde{q}_{2}, 0\right)$. To divide by a polynomial of the form $(q-\alpha)$ means to multiply by $(q-\alpha)^{-*}$, the $*$-reciprocal of $(q-\alpha)$, see [6] p.129, on the left or on the right. Assume to divide by multiplying on the left by $(q-\alpha)^{-*}$. It turns out that the polynomial $\left(q-q_{1}\right)^{-*} * w(q)=\left(q-q_{2}\right)$ does not vanish at $\tilde{q}_{2}$.

Despite these difficulties, it is possible to solve the problem of interpolating $n$ points over the quaternionic field. The problem has been addressed and solved in the general framework 
of division rings by Lam, see [4], who studied the Vandermonde matrix, its invertibility and interpolation. Other papers, in which similar results are obtained in the specific case of the algebra of quaternions, are [7] in which the quaternionic Vandermonde matrix and so the so-called double determinant is studied, and [8] which deals with the Cramer's rule.

In this paper, we will show that it is possible to construct in integral form the Lagrange interpolation polynomial $L_{n-1}(f)(q)$ of degree $n-1$ interpolating the values of a function slice regular in a ball $B(0, R)$ at the $n$ roots of the unit, i.e. the roots of $q^{n}-1=0$. Moreover, since a function $f$ slice regular admits power series expansion at the origin of the form $\sum_{k=0}^{\infty} q^{k} a_{k}$, we can construct the partial sum $s_{n-1}(f)(q)=\sum_{k=0}^{n-1} q^{k} a_{k}$ and we will prove the Walsh equiconvergence theorem (Theorem 3.6) and its converse (Theorem 3.9). We also treat, see Theorem 3.7, an extension of Walsh result. As in the complex case, we prove that our results are sharp. Our results are based on the fact that unilateral polynomials can be considered as slice regular functions, a class of functions defined over the quaternions which, despite the noncommutative setting, shares several properties of holomorphic functions of a complex variable.

\section{Preliminary results on quaternionic polynomials and slice regular functions}

Let us begin by recalling some terminology and preliminary results which will be useful in the sequel. An element in the skew field of quaternions $\mathbb{H}$ can be written as $q=x_{0}+$ $x_{1} \mathbf{i}+x_{2} \mathbf{j}+x_{3} \mathbf{k}$ where $x_{0}, \ldots, x_{3} \in \mathbb{R}$ and the imaginary units $\mathbf{i}, \mathbf{j}, \mathbf{k} \notin \mathbb{R}$ satisfy

$$
\mathbf{i}^{2}=\mathbf{j}^{2}=\mathbf{k}^{2}=-1, \mathbf{i j}=-\mathbf{j i}=\mathbf{k}, \mathbf{j k}=-\mathbf{k} \mathbf{j}=\mathbf{i}, \mathbf{k i}=-\mathbf{i k}=\mathbf{j}
$$

Given a quaternion $q=x_{0}+x_{1} \mathbf{i}+x_{2} \mathbf{j}+x_{3} \mathbf{k}$, its conjugate is defined as $\bar{q}=x_{0}-$ $x_{1} \mathbf{i}-x_{2} \mathbf{j}-x_{3} \mathbf{k}$ and its norm is $\|q\|=\sqrt{q \bar{q}}=\sqrt{x_{0}^{2}+x_{1}^{2}+x_{2}^{3}+x_{3}^{2}}$. Finally, the inverse of a nonzero quaternion $q$ is $q^{-1}=\bar{q} /\|q\|^{2}$.

We will denote by $\mathbb{S}$ the unit sphere of purely imaginary quaternion, i.e.

$$
\mathbb{S}=\left\{q=x_{1} \mathbf{i}+x_{2} \mathbf{j}+x_{3} \mathbf{k}, \text { such that } x_{1}^{2}+x_{2}^{2}+x_{3}^{3}=1\right\} .
$$

An element $I \in \mathbb{S}$ is such that $I^{2}=-1$ and for this reason the elements of $\mathbb{S}$ are also called imaginary units. For any $I \in \mathbb{S}$, we define $\mathbb{C}_{I}:=\{x+I y ; \mid x, y \in \mathbb{R}\}$. It is immediate that $\mathbb{C}_{I}$ can be identified with a complex plane. Any nonreal quaternion $q$ is uniquely associated to the element $I_{q} \in \mathbb{S}$ defined by $I_{q}:=\left(x_{1} \mathbf{i}+x_{2} \mathbf{j}+x_{3} \mathbf{k}\right) /\left\|x_{1} \mathbf{i}+x_{2} \mathbf{j}+x_{3} \mathbf{k}\right\|$ and, obviously, $q$ belongs to the complex plane $\mathbb{C}_{I_{q}}$. The real axis belongs to $\mathbb{C}_{I}$ for every $I \in \mathbb{S}$ and thus a real quaternion can be associated to any imaginary unit $I$. Sometimes, it will be useful to write a quaternion $q$ as $q=\operatorname{Re}(q)+I_{q}\|\operatorname{Im}(q)\|$ where $\operatorname{Re}(q)=x_{0}$ and $\operatorname{Im}(q)=x_{1} \mathbf{i}+x_{2} \mathbf{j}+x_{3} \mathbf{k}$.

In the literature, there are several notions extending the holomorphy to functions of a quaternionic variable. Here, we will consider the notion of slice regularity, see [9], since polynomials with quaternionic coefficients (on the right) belong to the class of (left) slice regular functions. Let us recall the following:

Definition 2.1 Let $U$ be an open set in $\mathbb{H}$ and $f: U \rightarrow \mathbb{H}$ real differentiable. $f$ is called left slice regular if for every $I \in \mathbb{S}$, its restriction $f_{I}$ to the complex plane $\mathbb{C}_{I}=\mathbb{R}+I \mathbb{R}$ passing through origin and containing $I$ and 1 satisfies 


$$
\bar{\partial}_{I} f(x+I y):=\frac{1}{2}\left(\frac{\partial}{\partial x}+I \frac{\partial}{\partial y}\right) f_{I}(x+I y)=0,
$$

on $U \bigcap \mathbb{C}_{I}$.

One can introduce the notion of right slice regular functions thus obtaining a different class of functions which, however, share properties analogous to those possessed by left slice regular functions. In this paper, we do not consider right slice regular functions, so for the sake of simplicity, we will refer to left slice regular functions as s-regular functions.

Let us now introduce the so-called (left) $I$-derivative of $f$ at a point $q=x+I y$ :

$$
\partial_{I} f_{I}(x+I y):=\frac{1}{2}\left(\frac{\partial}{\partial x} f_{I}(x+I y)-I \frac{\partial}{\partial y} f_{I}(x+I y)\right) .
$$

Using this notion, we can now introduce the following:

Definition 2.2 Let $U$ be an open set in $\mathbb{H}$, and let $f: U \rightarrow \mathbb{H}$ be a s-regular function. The slice derivative $\partial_{s} f$ of $f$ is defined by:

$$
\partial_{S}(f)(q)= \begin{cases}\partial_{I}(f)(q) & \text { if } q=x+I y, y \neq 0, \\ \frac{\partial f}{\partial x}(x) & \text { if } q=x \in \mathbb{R} .\end{cases}
$$

We have the following result characterizing s-regular functions on ball with centre at the origin (and more in general with centre at a real point):

Theorem 2.3 Let $B(0, R)=\{q \in \mathbb{H} ;\|q\|<R\}$ and let $q=x+$ Iy. A function $f: B(0, R) \rightarrow \mathbb{H}$ is s-regular if and only if it has a series representation of the form

$$
f(q)=\sum_{n=0}^{\infty} q^{n} \frac{1}{n !} \partial_{s}^{n} f(0)=\sum_{n=0}^{\infty} q^{n} \frac{1}{n !} \frac{\partial^{n} f}{\partial x^{n}}(0),
$$

convergent in $B(0, R)$.

Note that from this result, it easily follows that functions like the exponential, logartithm, sine, cosine and all the converging power series in the variable $q$ with real coefficients are s-regular functions. Another useful result which will be useful in the sequel is the Cauchy formula, see [6, Theorems 4.5.3, 4.5.4] and the definition of axially symmetric s-domain, see [6, Definitions 4.1.4, 4.3.1]. For our purposes, it is enough to know that balls in $\mathbb{H}$ are examples of axially symmetric s-domains.

THEOREM 2.4 Let $U \subseteq \mathbb{H}$ be an axially symmetric s-domain such that $\partial\left(U \cap \mathbb{C}_{I}\right)$ is union of a finite number of continuously differentiable Jordan curves, for every $I \in \mathbb{S}$. Let $f$ be a $s$-regular function on an open set containing $\bar{U}$ and, for any $I \in \mathbb{S}$, set $d s_{I}=-I d s$. Then, for every $q \in U$, we have:

$$
f(q)=\frac{1}{2 \pi} \int_{\partial\left(U \cap \mathbb{C}_{I}\right)}-\left(q^{2}-2 \operatorname{Re}[s] q+\|s\|^{2}\right)^{-1}(q-\bar{s}) d s_{I} f(s)
$$


where the value of the integral depends neither on $U$ nor on the imaginary unit $I \in \mathbb{S}$. Moreover,

$$
\partial_{s}^{n} f(q)=\frac{n !}{2 \pi} \int_{\partial\left(U \cap \mathbb{C}_{I}\right)}\left(q^{2}-2 s_{0} q+\|s\|^{2}\right)^{-n-1}(q-\bar{s})^{(n+1) *} d s_{I} f(s)
$$

where

$$
(q-\bar{s})^{n *}:=\sum_{k=0}^{n} \frac{n !}{(n-k) ! k !} q^{n-k} \bar{s}^{k}
$$

The kernel $-\left(q^{2}-2 \operatorname{Re}[s] q+\|s\|^{2}\right)^{-1}(q-\bar{s})$ is denoted in [6] by $S^{-1}(s, q)$; it is defined outside the set of zeros of $q^{2}-2 s_{0} q+\|s\|^{2}$ which will be described below. Since this kernel corresponds to the s-regular reciprocal of the function $S(s, q)=s-q$, in this paper we will denote it by $(s-q)^{-*}$ where, by this notation, we mean that we are taking the s-regular reciprocal with respect to the variable $q$.

As a corollary of Theorem 2.3, we immediately have that polynomials of the form $p(q)=\sum_{k=0}^{n} q^{k} a_{k}, a_{k} \in \mathbb{H}$, when considered as functions, are s-regular. Unilateral polynomials with quaternionic coefficients have been widely studied in the literature and, as it is well known, they can be multiplied with the $*$-product defined in (1). The $*$-product of two polynomials can be rewritten in terms of the pointwise product, see [4, Proposition 16.3, p.263]. Similarly, the $*$-product of two s-regular functions can be expressed as pointwise product, see [6, Proposition 4.3.22]:

Proposition 2.5 Let $\Omega \subseteq \mathbb{H}$ be an axially symmetric s-domain and let $f, g: \Omega \rightarrow \mathbb{H}$ be s-regular functions. Then if $f(q) \neq 0$

$$
f * g(q)=f(q) g\left(f(q)^{-1} q f(q)\right),
$$

for $q \in \Omega$. If $f(q)=0$ then $f * g(q)=0$.

Let us now turn the attention to unilateral polynomials in the quaternionic setting and their zeros, see [4]. In the sequel, we will denote by $\mathbb{H}[q]$ the quaternionic right linear space of polynomials with quaternionic coefficients on the right. To describe the zeros of polynomials in $\mathbb{H}[q]$, it is necessary to introduce an equivalence relation for quaternions: two elements $\alpha, \alpha^{\prime} \in \mathbb{H}$ are equivalent and we write $\alpha \sim \alpha^{\prime}$ if and only if $\operatorname{Re}(\alpha)=\operatorname{Re}\left(\alpha^{\prime}\right)$ and $\|\operatorname{Im}(\alpha)\|=\left\|\operatorname{Im}\left(\alpha^{\prime}\right)\right\|$. It is immediate that $\alpha \sim \alpha^{\prime}$ if and only if there exists $q \neq 0$ such that $q^{-1} \alpha q=\alpha^{\prime}$ or, in other words, if $\alpha^{\prime}$ can be obtained from $\alpha$ by a rotation. The set $[\alpha]$ is a sphere in $\mathbb{H}$ of the form $\operatorname{Re}(\alpha)+I\|\operatorname{Im}(\alpha)\|$ where $I \in \mathbb{S}$. When $\alpha \in \mathbb{R}$, the sphere reduces to the point $\alpha$, i.e. it has radius equal to 0 . We have the following well-known results, see $[4,10]$, that we collect in form of a Theorem for the reader's convenience:

Theorem 2.6

(1) A quaternion $\alpha$ is a zero of a (nonzero) polynomial $p \in \mathbb{H}[q]$ if and only if the polynomial $q-\alpha$ is a left divisor of $p(q)$.

(2) If $p(q)=\left(q-\alpha_{1}\right) * \cdots *\left(q-\alpha_{n}\right) \in \mathbb{H}[q]$, where $\alpha_{1}, \ldots, \alpha_{n} \in \mathbb{H}$, then $\alpha_{1}$ is a zero of $p$ and every other zero of $p$ is in the equivalence class of $\alpha_{i}, i=2, \ldots, n$. 
(3) If $p$ has two distinct zeros in an equivalence class $[\alpha]$, then all the elements in $[\alpha]$ are zeros of $p$.

When all the elements in $[\alpha]$ are zeros of $p$, we will say that $[\alpha]$ is a spherical zero of $p$. It is easy to verify that the elements belonging to the equivalence class $[\alpha]$ are roots of the following polynomial (sometimes called the minimal polynomial or companion polynomial of the equivalence class $[\alpha])$ :

$$
Q_{\alpha}(q)=(q-\alpha) *(q-\bar{\alpha})=q^{2}-2 \operatorname{Re}(\alpha) q+\|\alpha\|^{2} .
$$

We also have (see $[3,11])$ :

Proposition 2.7 We have $Q_{\alpha}(q)=Q_{\alpha^{\prime}}(q)$ if and only if $[\alpha]=\left[\alpha^{\prime}\right]$. If $Q_{\alpha}$ divides a polynomial $p(q)$ then $p(\lambda)=0$ for every $\lambda \in[\alpha]$. Otherwise, at most one element in $[\alpha]$ is a zero of $p$.

From this discussion, we immediately have:

PROposition 2.8 The quaternionic equation $q^{n}-1=0$ has the real zero $q=1$ and $k$ spherical zeros if $n=2 k+1$, while it has the real zeros $q= \pm 1$ and $k-1$ spherical zeros if $n=2 k$.

Proof To solve the equation $q^{n}-1=0$ over the quaternions, we first solve $z^{n}-1=0$ in the complex field. This amounts to look for the solutions of the quaternionic equations belonging to a complex plane $\mathbb{C}_{I}$. As it is well known, the complex equation has the real zero +1 and pairs of conjugate roots if $n=2 k+1$; it has zeros \pm 1 and $k-1$ pairs of conjugate zeros if $n=2 k$. By Theorem 2.6, each pair of conjugate roots corresponds to a spherical zero.

From this proposition, it follows that $q^{n}-1$ has an infinite number of zeros so, in principle, we have an infinite number of points where to interpolate. However, the zeros belong to a finite number of spheres and this will be enough to guarantee that we can interpolate at a finite number of points. In fact, we have the following result (see [6, Corollary 4.3.6]):

PROPOSITION 2.9 The values of an s-regular function $f$ and, in particular, of a polynomial at two points belonging to a same equivalence class $[\alpha]$ determine the values of $f$ at all the other points in $[\alpha]$.

\subsection{The interpolation problem}

Let us now come to the problem of interpolating the values of a s-regular function $f$ at the roots of the unity $q^{n}-1=0$ over the quaternions. From Proposition 2.9, we deduce that it will mean to assign the $n$ values of the function $f$ at $n$ points corresponding to:

- the real root +1 of the equation $q^{n}-1$ and $k$ pairs of points (not necessarily conjugated) belonging to a sphere of roots $\left[\alpha_{j}\right], j=1, \ldots, k$, if $n=2 k+1$; 
- the real roots \pm 1 of the equation $q^{n}-1$ and $k-1$ pairs of points (not necessarily conjugated) belonging to a sphere of roots $\left[\alpha_{j}\right], j=1, \ldots, k-1$, if $n=2 k$.

As in the complex case, to look for the polynomial of degree $p(q)=\sum_{j=0}^{n-1} q^{j} a_{j}$ interpolating $\left(q_{i}, w_{i}\right), i=1, \ldots, n$ leads to the system:

$$
\begin{gathered}
a_{0}+q_{1} a_{1}+\cdots+q_{1}^{n-1} a_{n-1}=w_{1} \\
\cdots \\
a_{0}+q_{n} a_{1}+\cdots+q_{n}^{n-1} a_{n-1}=w_{n}
\end{gathered}
$$

which can be written as

$$
V\left(q_{1}, \ldots, q_{n}\right) \underline{a}=\underline{w},
$$

where $\underline{a}=\left[a_{0}, \ldots, a_{n-1}\right]^{T}, \underline{w}=\left[w_{0}, \ldots, w_{n}\right]^{T}$ and whose matrix of coefficients is the Vandermonde matrix:

$$
V\left(q_{1}, \ldots, q_{n}\right)=\left[\begin{array}{llll}
1 & q_{1} & \cdots & q_{1}^{n-1} \\
& & \cdots & \\
1 & q_{n} & \cdots & q_{n}^{n-1}
\end{array}\right]
$$

The Vandermonde matrix in division rings has been studied in [12] and [13] in which a sufficient condition in order to have the invertibility of $V\left(q_{1}, \ldots, q_{n}\right)$ and the unique solution to the interpolation problem $f\left(q_{i}\right)=w_{i}, i=1, \ldots, n$ is given, see [12, Corollary 24]. The condition, in our case, can be expressed as follows:

THEOREM 2.10 Given $q_{1}, \ldots, q_{n} \in \mathbb{H}$ such that no three of them belong to the same equivalence class, the Vandermonde matrix $V\left(q_{1}, \ldots, q_{n}\right)$ is invertible and for any $w_{1}, \ldots, w_{n}$ there exists a unique solution to the interpolation problem (6).

In the paper [7], the authors have studied the specific case of quaternions reobtaining the result in [12, Corollary 24] with a different method. In the language of [7], the so-called double determinant of the Vandermonde matrix $V\left(q_{1}, \ldots, q_{n}\right)$ is nonzero if and only if no three of the $q_{j}$ 's are in the same equivalence class, see [7, Theorem 4.1]. This is the necessary and sufficient condition in order to have a unique solution of (6) by virtue of the analogue of Cramer's rule proven in [8, Theorem 2.3]. In recent times, the Vandermonde matrix and the interpolation problem have been considered also in [14], while an interpolation problem in the Hardy space of the unit ball has been considered in [15].

Remark 2.11 Thus, we have that, in the case of interpolation at the roots of unity, the interpolation problem admits unique solution if we assign the values at the real root(s) of $q^{n}-1=0$ and at two elements in each equivalence class $[\alpha]$ which is a root of the equation (recall that, by Proposition 2.9, the obtained interpolation polynomial will have uniquely determined values at all points of $[\alpha])$.

\section{Walsh equiconvergence theorem in quaternionic setting}

Let us start by giving a definition which, as we shall see, will correspond to the analogue of the Lagrange interpolation polynomial at the roots of the unity over the quaternions: 
Definition 3.1 Let $f: B(0 ; \rho) \rightarrow \mathbb{H}$ be (left) s-regular and let $\Gamma_{R, I}=\{u \in \mathbb{H}$, $u=x+I y ;\|u\|=R\}, 1<R<\rho$, in other words, $\Gamma_{R, I}$ is the intersection of the ball with centre at the origin and radius $R$ with the complex plane $\mathbb{C}_{I}$. We define

$$
L_{n-1}(f)(q)=\frac{1}{2 \pi} \int_{\Gamma_{R, I}}(\xi-q)^{-*} *\left(\xi^{n}-q^{n}\right)\left(\xi^{n}-1\right)^{-1} d \xi_{I} f(\xi), q \in B(0 ; R), d \xi_{I}=d \xi / I,
$$

where the $*$-product is taken with respect to $q$. Note that formula (7) seems to depend on the plane on which the integral is computed. However this is not the case by virtue of the following result:

Proposition 3.2 The definition of $L_{n-1}(f)(q)$ does not depend on the choice of $I \in \mathbb{S}$.

Proof Let $I$ arbitrary in $\mathbb{S}$ and let $J \in \mathbb{S}$ be such that $I \perp J$. Then by the Splitting Lemma for s-regular functions, see [9] or [6], the restriction of the s-regular function $f$ to $\mathbb{C}_{I}$ can be written as $f_{\mid \mathbb{C}_{I}}(\xi)=F(\xi)+G(\xi) J$ where $F, G: B(0, \rho) \cap \mathbb{C}_{I} \rightarrow \mathbb{C}_{I}$ are holomorphic functions of the complex variable $\xi$. For the holomorphic function $F$ (resp. $G$ ) the Lagrange polynomials can be obtained as

$$
L_{n-1}(F)(z)=\frac{1}{2 \pi} \int_{\Gamma_{R, I}}(\xi-z)^{-1}\left(\xi^{n}-z^{n}\right)\left(\xi^{n}-1\right)^{-1} d \xi_{I} F(\xi),
$$

so we get

$$
L_{n-1}(F+G J)(z)=\frac{1}{2 \pi} \int_{\Gamma_{R, I}}(\xi-z)^{-1}\left(\xi^{n}-z^{n}\right)\left(\xi^{n}-1\right)^{-1} d \xi_{I}(F(\xi)+G(\xi) J),
$$

i.e.

$$
L_{n-1}\left(f_{\mid \mathbb{C}_{I}}\right)(z)=\frac{1}{2 \pi} \int_{\Gamma_{R, I}}(\xi-z)^{-1}\left(\xi^{n}-z^{n}\right)\left(\xi^{n}-1\right)^{-1} d \xi_{I}\left(f_{\mid \mathbb{C}_{I}}(\xi)\right),
$$

where $z \in B(0 ; R) \cap \mathbb{C}_{I}, d \xi_{I}=d \xi / I$. By the Representation Formula, see [6, Theorem 4.3.2], the value of the (s-regular) polynomial $L_{n-1}\left(f_{\mid \mathbb{C}_{I}}\right)(z)$ at any other point of $q=x+J y \in B(0 ; R)$ can be computed as

$$
L_{n-1}(f)(q)=\frac{1}{2}\left[L_{n-1}\left((1+J I) f_{\mid \mathbb{C}_{I}}\right)(z)+(1-J I) L_{n-1}\left(f_{\mid \mathbb{C}_{I}}\right)(z)\right]
$$

and since

$\frac{1}{2}\left[(1+J I)(\xi-z)^{-1}\left(\xi^{n}-z^{n}\right)+(1-J I)(\xi-z)^{-1}\left(\xi^{n}-z^{n}\right)\right]=(\xi-q)^{-*} *\left(\xi^{n}-q^{n}\right)$

we get formula (7).

Remark 3.3 Another way to justify that the integral (7) does not depend on $I \in \mathbb{S}$ is the fact that $L_{n-1}(f)(q)$ is a polynomial of degree $n-1$ and its uniqueness, see Theorem 2.10, guarantees that the definition does not depend on the choice made.

We now show that $L_{n-1}(f)$ is a polynomial of degree $n-1$ interpolating $f$ on the roots of $q^{n}-1=0$. As we discussed in Section 2.1, a polynomial interpolating $n$ roots of the unity, not three of them on the same equivalence class, in reality interpolates $f$ at all 
the possible solutions to the equation $q^{n}-1=0$. To state next result, we denote the roots of $q^{n}-1=0$ at which we are interpolating as $q_{k, n}=\cos (2 k \pi / n)+J_{n, k} \sin (2 k \pi / n)$, $k=0,1, \ldots, n-1, J_{n, k} \in \mathbb{S}$.

Proposition 3.4 Given the s-regular function $f: B(0 ; \rho) \rightarrow \mathbb{H}$, then $L_{n-1}(f)$ as in (7) is a polynomial of degree $n-1$ in the variable $q$ and

$$
L_{n-1}(f)\left(q_{k, n}\right)=f\left(q_{k, n}\right), \quad \text { for all } k=0,1, \ldots, n-1,
$$

where $q_{k, n}=\cos (2 k \pi / n)+J_{n, k} \sin (2 k \pi / n)$, for all $J_{n, k} \in \mathbb{S}$ i.e. $L_{n-1}(f)$ interpolates $f$ at the roots of the unity.

Proof First of all, we note that the polynomial $\left(\xi^{n}-q^{n}\right)$ vanishes when $\xi=q$, thus $(\xi-q)$ is a left factor and we can write $\left(\xi^{n}-q^{n}\right)=(\xi-q) * P_{n-1}(\xi, q)$ where $P_{n-1}(\xi, q)$ has degree $n-1$ in $q$. So we have

$$
\begin{aligned}
L_{n-1}(f)(q) & =\frac{1}{2 \pi} \int_{\Gamma_{R, I}}(\xi-q)^{-*} *(\xi-q) * P_{n-1}(\xi, q)\left(\xi^{n}-1\right)^{-1} d \xi_{I} f(\xi) \\
& =\frac{1}{2 \pi} \int_{\Gamma_{R, I}} P_{n-1}(\xi, q)\left(\xi^{n}-1\right)^{-1} d \xi_{I} f(\xi)
\end{aligned}
$$

and $L_{n-1}(f)(q)$ is an s-regular polynomial of degree $n-1$ in $q$ because so is $P_{n-1}(\xi, q)$. Let us consider now the root of the unity $q_{k, n}=\cos (2 k \pi / n)+J_{n, k} \sin (2 k \pi / n)$ and let us compute $L_{n-1}(f)\left(q_{k, n}\right)$. Recalling that the evaluation is not a homomorphism with respect to the $*$-product, we cannot substitute $q_{n, k}$ in place of $q$ in the expression $(\xi-q)^{-*} *\left(\xi^{n}-q^{n}\right)$. However, (5) gives

$$
(\xi-q)^{-*} *\left(\xi^{n}-q^{n}\right)=(\xi-q)^{-*}\left(\xi^{n}-\tilde{q}^{n}\right),
$$

where $\tilde{q}=f(q)^{-1} q f(q)$ with $f(q)=(\xi-q)^{-*}$. Then $\tilde{q}^{n}=f(q)^{-1} q^{n} f(q)$ and and so $\left(\tilde{q}^{n}\right)_{\mid q_{k, n}}=1$, thus we have:

$$
\left.\left((\xi-q)^{-*} *\left(\xi^{n}-q^{n}\right)\right)\right|_{q=q_{k, n}}\left(\xi-q_{k, n}\right)^{-*}\left(\xi^{n}-1\right) .
$$

Note that, in this case, the result follows also by direct computation in fact:

$$
\begin{aligned}
(\xi-q)^{-*} *\left(\xi^{n}-q^{n}\right) & =\left(q^{2}-2 \operatorname{Re}(\xi) q+\|\xi\|^{2}\right)^{-1}(\bar{\xi}-q) *\left(\xi^{n}-q^{n}\right) \\
& =\left(q^{2}-2 \operatorname{Re}(\xi) q+\|\xi\|^{2}\right)^{-1}\left(\bar{\xi} \xi^{n}-q \xi^{n}-q^{n} \bar{\xi}+q^{n+1}\right)
\end{aligned}
$$

thus

$$
\begin{aligned}
\left.\left((\xi-q)^{-*} *\left(\xi^{n}-q^{n}\right)\right)\right|_{q=q_{k, n}} \\
\quad=\left(q_{k, n}^{2}-2 \operatorname{Re}(\xi) q_{k, n}+\|\xi\|^{2}\right)^{-1}\left(\bar{\xi} \xi^{n}-q_{k, n} \xi^{n}-q_{k, n}^{n} \bar{\xi}+q_{k, n}^{n+1}\right) \\
\quad=\left(q_{k, n}^{2}-2 \operatorname{Re}(\xi) q_{k, n}+\|\xi\|^{2}\right)^{-1}\left(\bar{\xi} \xi^{n}-q_{k, n} \xi^{n}-\bar{\xi}+q_{k, n}\right) \\
\quad=\left(q_{k, n}^{2}-2 \operatorname{Re}(\xi) q_{k, n}+\|\xi\|^{2}\right)^{-1}\left(\bar{\xi}-q_{k, n}\right)\left(\xi^{n}-1\right) \\
\quad=\left(\xi-q_{k, n}\right)^{-*}\left(\xi^{n}-1\right) .
\end{aligned}
$$


Using these calculations, and recalling that $L_{n-1}(f)$ does not depend on the plane on which we integrate, we finally have:

$$
\begin{aligned}
L_{n-1}(f)\left(q_{k, n}\right) & =\left.\frac{1}{2 \pi} \int_{\Gamma_{R, J_{n, k}}}\left((\xi-q)^{-*} *\left(\xi^{n}-q^{n}\right)\right)\right|_{q=q_{k, n}}\left(\xi^{n}-1\right)^{-1} d \xi_{J_{n, k}} f(\xi) \\
& =\frac{1}{2 \pi} \int_{\Gamma_{R, J_{n, k}}}\left(\xi-q_{k, n}\right)^{-*}\left(\xi^{n}-1\right)\left(\xi^{n}-1\right)^{-1} d \xi_{J_{n, k}} f(\xi) \\
& =\frac{1}{2 \pi} \int_{\Gamma_{R, J_{n, k}}}\left(\xi-q_{k, n}\right)^{-*} d \xi_{J_{n, k}} f(\xi)=f\left(q_{k, n}\right)
\end{aligned}
$$

where the last equality follows from the Cauchy formula (3).

We point out that in the special case in which the function $f$ s-regular on $B(\rho, 0)$ is quaternionic intrinsic, i.e. $f(\bar{q})=\overline{f(q)}$, then by the Splitting Lemma we have that its restriction to a complex plane $\mathbb{C}_{I}$ is of the form $f_{\mid \mathbb{C}_{I}}(\zeta)=F(\zeta)$ where $F$ is a holomorphic function on $B(\rho, 0) \cap \mathbb{C}_{I}$. Reasoning as in the proof of Proposition 3.2, we have that the Lagrange polynomial $L_{n-1}\left(f_{\mid \mathbb{C}_{I}}\right)(\zeta)=L_{n-1}(F)(\zeta)$ of the holomorphic function $F$ is of the form (2) where $z_{k}$ are the roots of the unity belonging to the complex plane $\mathbb{C}_{I}$. By the representation formula, this polynomial extends to a quaternionic polynomial of the form (2).

To our purposes, we also need an integral representation for the partial sum $s_{n-1}(f)(q)$ of the Taylor series at the origin of function s-regular in a ball centred at the origin. If $f(q)=\sum_{k=0}^{\infty} q^{k} a_{k}$, we set $s_{n-1}(f)(q):=\sum_{k=0}^{n-1} q^{k} a_{k}$ where $a_{k}=\frac{1}{k !} \partial_{s}^{k} f(0)$. We have the following result:

Proposition 3.5 Let $f: B(0 ; \rho) \rightarrow \mathbb{H}$ be s-regular and let $\Gamma_{R, I}=\{u \in \mathbb{H}, u=$ $x+I y ;\|u\|=R\}, R<\rho$. We can write

$$
s_{n-1}(f)(q)=\frac{1}{2 \pi} \int_{\Gamma_{R, I}}(\xi-q)^{-*} *\left(\xi^{n}-q^{n}\right) \xi^{-n} d \xi_{I} f(\xi),
$$

where the integral does not depend on the choice of $I \in \mathbb{S}$.

Proof When $\|q\|<\|\xi\|$, we can write, see [6] p.53:

$$
\begin{aligned}
(\xi-q)^{-*} & =\sum_{n=0}^{\infty} q^{n} \xi^{-n-1}=\left(\sum_{k=0}^{n-1} q^{k} \xi^{-k}+\sum_{k=n}^{\infty} q^{k} \xi^{-k}\right) \xi^{-1} \\
& =\left(\sum_{k=0}^{n-1} q^{k} \xi^{-k}+q^{n}\left(\sum_{k=0}^{\infty} q^{k} \xi^{-k-1}\right) \xi^{-n+1}\right) \xi^{-1} \\
& =\left(\sum_{k=0}^{n-1} q^{k} \xi^{-k}+q^{n}(\xi-q)^{-*} \xi^{-n+1}\right) \xi^{-1}
\end{aligned}
$$

Note that (9) is valid outside the singularities of $(\xi-q)^{-*}$ and not only in the ball $\|q\|<\|\xi\|$. 
By using the Cauchy integral formula, which is independent of the choice of $I \in \mathbb{S}$, and (9) we get

$$
\begin{aligned}
f(q) & =\frac{1}{2 \pi} \int_{\Gamma_{R, I}}(\xi-q)^{-*} d \xi_{I} f(\xi) \\
& =\sum_{k=0}^{n-1} q^{k}\left(\frac{1}{2 \pi} \int_{\Gamma_{R, I}} \xi^{-k-1} d \xi_{I} f(\xi)\right)+\frac{1}{2 \pi} \int_{\Gamma_{R, I}} q^{n}(\xi-q)^{-*} \xi^{-n} d \xi_{I} f(\xi) \\
& =s_{n-1}(f)(z)+\frac{1}{2 \pi} \int_{\Gamma_{R, I}} q^{n}(\xi-q)^{-*} \xi^{-n} d \xi_{I} f(\xi),
\end{aligned}
$$

where we used the fact that (see Theorem 2.4):

$$
\frac{1}{k !} \partial_{s}^{k} f(0)=\frac{1}{2 \pi} \int_{\Gamma_{R, I}} \xi^{-k-1} d \xi_{I} f(\xi)
$$

The equality (10) yields

$$
s_{n-1}(f)(q)=\frac{1}{2 \pi} \int_{\Gamma_{R, I}}(\xi-q)^{-*} d \xi_{I} f(\xi)-\frac{1}{2 \pi} \int_{\Gamma_{R, I}} q^{n}(\xi-q)^{-*} \xi^{-n} d \xi_{I} f(\xi) .
$$

The function $(\xi-q)^{-*}-q^{n}(\xi-q)^{-*} \xi^{-n}$, which is s-regular in the variable $q$, can be written as

$$
\begin{aligned}
(\xi-q)^{-*}-q^{n}(\xi-q)^{-*} \xi^{-n} & =(\xi-q)^{-*}-(\xi-q)^{-*} * q^{n} \xi^{-n} \\
& =(\xi-q)^{-*} *\left(1-q^{n} \xi^{-n}\right) \\
& =(\xi-q)^{-*} *\left(\xi^{n}-q^{n}\right) \xi^{-n},
\end{aligned}
$$

and so we obtain the statement.

We are now ready to prove the Walsh equiconvergence theorem in the quaternionic setting.

Theorem 3.6 (Walsh equiconvergence) Let $\rho>1$ and $f: B(0 ; \rho) \rightarrow \mathbb{H}$ be s-regular, i.e. $f(q)=\sum_{k=0}^{\infty} q^{k} a_{k}$ with $a_{k} \in \mathbb{H}$. Then

(i) $L_{n-1}(f)$ given by (7) converges uniformly and geometrically to $f$ in any closed ball $\overline{B(0 ; R)}$ with $1<R<\rho$;

(ii) $\lim _{n \rightarrow \infty}\left[s_{n-1}(f)(q)-L_{n-1}(f)(q)\right]=0$, for all $\|q\|<\rho^{2}$, the convergence being again uniform and with geometric rate of convergence in any $\overline{B(0 ; R)}$ with $1<R<\rho^{2}$;

(iii) the result is the best possible, in the sense that for the s-regular function $g$ : $B(0 ; \rho) \rightarrow \mathbb{H}$, given by $g(q)=(q-\rho)^{-1}$, for $q=\rho^{2}$ we have $\lim _{n \rightarrow \infty}\left[s_{n-1}(g)(q)-\right.$ $\left.L_{n-1}(g)(q)\right] \neq 0$. 
Proof (i) Taking into account the Cauchy integral formula, see Theorem 2.4, we get

$$
\begin{array}{r}
f(q)-L_{n-1}(f)(q)=\frac{1}{2 \pi} \int_{\Gamma_{R, I}}(\xi-q)^{-*} *\left[1-\left(\xi^{n}-q^{n}\right)\left(\xi^{n}-1\right)^{-1}\right] d \xi_{I} f(\xi) \\
q \in B(0 ; R) .
\end{array}
$$

Denoting $A:=1-\left(\xi^{n}-q^{n}\right)\left(\xi^{n}-1\right)^{-1}$, we get $1-A=\left(\xi^{n}-q^{n}\right)\left(\xi^{n}-1\right)^{-1}$, which implies $(1-A)\left(\xi^{n}-1\right)=\xi^{n}-q^{n}$. A simple calculation implies $A=\left(q^{n}-1\right)\left(\xi^{n}-1\right)^{-1}$, which replaced in the integral above gives

$$
f(q)-L_{n-1}(f)(q)=\frac{1}{2 \pi} \int_{\Gamma_{R, I_{q}}}(\xi-q)^{-*} *\left(q^{n}-1\right)\left(\xi^{n}-1\right)^{-1} d \xi_{I} f(\xi) .
$$

Denote $M=\max \{\|f(\xi)\| ; \xi \in \partial B(0 ; R)\}<\infty$ (see the maximum modulus principle [9]) and take an arbitrary $1<\mu<R$. For all $\|\xi\|=R$ and $\|q\| \leq \mu$ we immediately get $\left\|\xi^{n}-1\right\| \geq R^{n}-1$ and $\left\|q^{n}-1\right\| \leq \mu^{n}+1$. Recalling (5) we obtain that $\left\|(\xi-q)^{-*}\right\|=$ $\left\|(\xi-\tilde{q})^{-1}\right\| \geq|\|\xi\|-\|q\||=R-\|q\| \geq R-\mu$ which implies

$$
\left\|(\xi-q)^{-*} *\left(q^{n}-1\right)\left(\xi^{n}-1\right)^{-1}\right\|=\left\|(\xi-\tilde{q})^{-1}\right\|\left\|\hat{q}^{n}-1\right\|\left\|\left(\xi^{n}-1\right)^{-1}\right\|
$$

where $\tilde{q}, \hat{q}$ are elements in the sphere defined by $q$ according to (5) thus $\|\tilde{q}\|=\|\hat{q}\|=\|q\|$ and so

$$
\left\|(\xi-\tilde{q})^{-1}\right\|\left\|\hat{q}^{n}-1\right\|\left\|\left(\xi^{n}-1\right)^{-1}\right\| \leq \frac{\mu^{n}+1}{\left(R^{n}-1\right)(R-\mu)} \leq \frac{4}{R-\mu} \cdot\left(\frac{\mu}{R}\right)^{n} .
$$

Therefore

$$
\left\|f(q)-L_{n-1}(f)(q)\right\| \leq M R \cdot \frac{\mu^{n}+1}{\left(R^{n}-1\right)(R-\mu)} \leq C\left(\frac{\mu}{R}\right)^{n}, \text { for all }|q| \leq \mu<R,
$$

with $C=\frac{4 M R}{R-\mu}$ independent of $n$ and $q$.

As $1<R<\rho$ was arbitrary chosen, it follows the uniform convergence with geometric rate $\left(\frac{\mu}{R}\right)^{n}$, of $L_{n-1}(f)$ to $f$ in any closed disc included in $\mathbb{D}_{\rho}$, which proves the first part of the theorem.

(ii) For the second part of the theorem, we consider for any $q \in B(0, R)$ the difference

$$
\begin{aligned}
L_{n-1}(f)(q)-s_{n-1}(f)(q) & \\
= & \frac{1}{2 \pi} \int_{\Gamma_{R, I}}(\xi-q)^{-*} *\left(\xi^{n}-q^{n}\right)\left[\left(\xi^{n}-1\right)^{-1}-\xi^{-n}\right] d \xi_{I} f(\xi) \\
& =\frac{1}{2 \pi} \int_{\Gamma_{R, I}}(\xi-q)^{-*} *\left(\xi^{n}-q^{n}\right)\left(\xi^{n}-1\right)^{-1} \xi^{-n} d \xi_{I} f(\xi) .
\end{aligned}
$$

Since $(\xi-q)$ is a left factor of $\xi^{n}-q^{n}$, the integrand at the right-hand side is s-regular for all (finite) values of $q$ and in particular for $\|q\|=\mu<\rho^{2}$. The estimate

$$
\left\|(\xi-q)^{-*} *\left(\xi^{n}-q^{n}\right)\left(\xi^{n}-1\right)^{-1} \xi^{-n}\right\| \leq \frac{R^{n}+\mu^{n}}{(R-\mu)\left(R^{n}-1\right) R^{n}}
$$


implies

$$
\begin{aligned}
\left\|L_{n-1}(f)(q)-s_{n-1}(f)(q)\right\| & \leq \frac{M R\left(R^{n}+\mu^{n}\right)}{R^{n}\left(R^{n}-1\right)(R-\mu)} \leq \frac{2 M R}{R-\mu} \cdot\left(\frac{R^{n}+\mu^{n}}{R^{2 n}}\right) \\
& =C\left(\frac{1}{R^{n}}+\left(\frac{\mu}{R^{2}}\right)^{n}\right),
\end{aligned}
$$

with $C=\frac{2 M R}{R-\mu}$ and the last term in the above inequality tending to zero (as $n \rightarrow \infty$ ) for any $\mu<R^{2}$, which proves the second part of the theorem too.

(iii) For the the third part of the theorem, let $g(q)=(q-\rho)^{-*}=(q-\rho)^{-1}, q \in B(0 ; 1)$. In this case, for $\|q\|<\rho$, by [6], p.53 (see also the proof of Proposition 3.4), we can write

$$
g(q)=-(\rho-q)^{-1}=-\frac{1}{\rho} \cdot \sum_{k=0}^{\infty}\left(\frac{q}{\rho}\right)^{k}
$$

and obviously that the partial sum $s_{n-1}(g)(q)$ for this $f$ will be

$$
s_{n-1}(g)(q)=-\frac{1}{\rho} \cdot \sum_{k=0}^{n-1}\left(\frac{q}{\rho}\right)^{k},
$$

which immediately implies that

$$
\begin{gathered}
f(q)-s_{n-1}(g)(q) \\
=-\frac{1}{\rho} \cdot \sum_{k=n}^{+\infty}\left(\frac{q}{\rho}\right)^{k}=\sum_{j=0}^{+\infty}\left(\frac{q}{\rho}\right)^{j} \cdot-\frac{1}{\rho} \cdot \frac{q^{n}}{\rho^{n}}=\left(1-\frac{q}{\rho}\right)^{-1} \cdot \frac{-q^{n}}{\rho^{n+1}}=(q-\rho)^{-1} \cdot \frac{q^{n}}{\rho^{n}} .
\end{gathered}
$$

On the other hand, by the above formula, we get

$$
g(q)-L_{n-1}(g)(q)=\frac{1}{2 \pi} \int_{\Gamma_{R, I}}(\xi-q)^{-*}\left(q^{n}-1\right)\left(\xi^{n}-1\right)^{-1}(-I) d \xi \cdot(\xi-\rho)^{-1} .
$$

Since the integral formula does not depend on the choice of the complex plane $\mathbb{C}_{I}$, we can choose to integrate on the plane $\mathbb{C}_{I_{q}}$. The integrand at the right-hand side can be viewed as an integral in the complex plane $\mathbb{C}_{I_{q}}$ and therefore formally calculated exactly as in the complex case (note that this is true because $\rho \in \mathbb{R}$ ). Consequently, taking into account the computations in the complex case (see [5], p.154), we get

$$
g(q)-L_{n-1}(g)(q)=(q-\rho)^{-1}\left(q^{n}-1\right)\left(\rho^{n}-1\right)^{-1} .
$$

Collecting the results, it follows

$$
L_{n-1}(g)(q)-s_{n-1}(g)(q)=(q-\rho)^{-1} \cdot \frac{\rho^{n}-q^{n}}{\left(\rho^{n}-1\right) \rho^{n}} .
$$

Now, taking $q=\rho^{2}$, we easily get $L_{n-1}(g)(q)-s_{n-1}(g)(q)=\frac{1}{\rho-\rho^{2}}$, which obviously does not approach zero.

As in the complex case, many extensions of the Walsh's theorem could be given. The result below is a generalization of [16, Theorem 1] (for the points (i) and (ii)) and of [2, Theorem 1] (for the point (iii)). 
THEOREM 3.7 Let $\rho>1$ and $f: B(0 ; \rho) \rightarrow \mathbb{H}$ be s-regular, i.e. of the form $f(q)=$ $\sum_{k=0}^{\infty} q^{k} a_{k}$, with $a_{k} \in \mathbb{H}$. For $l \in \mathbb{N}$ and $j=0,1, \ldots, l-1$, let us define the polynomials $P_{n-1, j}(f)(q)=\sum_{k=0}^{n-1} q^{k} a_{k+j n}$ and $S_{n-1, l}(f)(q)=\sum_{j=0}^{l-1} P_{n-1, j}(f)(q)$.

(i) We have

$$
\lim _{n \rightarrow \infty}\left[L_{n-1}(f)(q)-S_{n-1, l}(f)(q)\right]=0, \text { for all }\|q\|<\rho^{l+1},
$$

the convergence being uniform and with geometric rate of convergence in any closed ball $\overline{B(0 ; R)}$ with $1<R<\rho^{l+1}$;

(ii) the convergence is the best possible, in the sense that for $g(q)=(q-\rho)^{-1}$ and for $q=\rho^{l+1}$, we have $\lim _{n \rightarrow \infty}\left[L_{n-1}(g)(q)-S_{n-1, l}(g)(q)\right] \neq 0$;

(iii) if $f$ has a singularityon $\|q\|=\rho$, then the sequence $\left(L_{n-1}(f)(q)-S_{n-1, l}(f)(q)\right)_{n \in \mathbb{N}}$ can be bounded in at most $l$ distinct points $q_{k}, k=1, \ldots, l$, such that any three of them do not belong to the same sphere and satisfy $\left\|q_{k}\right\|>\rho^{l+1}, k=1, \ldots, l$.

Proof (i) Taking into account formula (11), by simple calculation (similar to those in the complex case, see the proof of Theorem 1 in [16]), we easily arrive at the formula

$$
\begin{aligned}
P_{n-1, j}(f)(q) & =\frac{1}{2 \pi} \int_{\Gamma_{R, I_{q}}}(\xi-q)^{-*} *\left(\xi^{n}-q^{n}\right)\left(\xi^{n(j+1)}\right)^{-1} d \xi_{I_{q}} f(\xi), \\
j & =0,1, \ldots,
\end{aligned}
$$

Therefore, taking into account the formula in Definition 3.1, for each integer $l \geq 1$, we obtain

$$
\begin{aligned}
& L_{n-1}(f)(q)-S_{n-1, l}(f)(q) \\
& =\frac{1}{2 \pi} \int_{\Gamma_{R, I_{q}}}(\xi-q)^{-*}\left(\xi^{n}-q^{n}\right)\left(\xi^{n}-1\right)^{-1} d \xi_{I_{q}} f(\xi) \\
& \quad-\frac{1}{2 \pi} \int_{\Gamma_{R, I_{q}}}(\xi-q)^{-*} *\left(\xi^{n}-q^{n}\right) \sum_{j=0}^{l-1}\left(\xi^{n(j+1)}\right)^{-1} d \xi_{I_{q}} f(\xi) \\
& =\frac{1}{2 \pi} \int_{\Gamma_{R, I_{q}}}(\xi-q)^{-*} *\left(\xi^{n}-q^{n}\right)\left[\left(\xi^{n}-1\right)^{-1}-\sum_{j=0}^{l-1}\left(\xi^{n(j+1)}\right)^{-1}\right] d \xi_{I_{q}} f(\xi) .
\end{aligned}
$$

By simple properties of the powers of quaternions, we get

$$
\left(\xi^{n}-1\right)^{-1}-\sum_{j=0}^{l-1}\left(\xi^{n(j+1)}\right)^{-1}=\left(\xi^{n}-1\right)^{-1}\left(\xi^{n l}\right)^{-1}
$$

and therefore

$$
\begin{aligned}
& L_{n-1}(f)(q)-S_{n-1, l}(f)(q) \\
& =\frac{1}{2 \pi} \int_{\Gamma_{R, I_{q}}}(\xi-q)^{-*} *\left(\xi^{n}-q^{n}\right)\left(\xi^{n}-1\right)^{-1}\left(\xi^{n l}\right)^{-1} d \xi_{I_{q}} f(\xi) .
\end{aligned}
$$


Let $1<\mu<R,\|t\|=R,\|q\| \leq \mu$ and denote $M=\max \{\|f(q)\| ;\|q\|=R\}$. Reasoning exactly as in the proof of Theorem 3.6, we get

$$
\left\|(\xi-q)^{-*} *\left(\xi^{n}-q^{n}\right)\left(\xi^{n}-1\right)^{-1}\left(\xi^{n l}\right)^{-1} f(\xi)\right\| \leq \frac{M\left(R^{n}+\mu^{n}\right)}{R^{n l}\left(R^{n}-1\right)(R-\mu)},
$$

which implies

$$
\begin{aligned}
\left\|L_{n-1}(f)(q)-S_{n-1, l}(f)(q)\right\| & \leq \frac{M R\left(R^{n}+\mu^{n}\right)}{R^{n l}\left(R^{n}-1\right)(R-\mu)} \leq \frac{2 M R}{R-\mu} \cdot\left(\frac{R^{n}+\mu^{n}}{R^{n(l+1)}}\right) \\
& =C\left(\frac{1}{R^{n l}}+\left(\frac{\mu}{R^{l+1}}\right)^{n}\right),
\end{aligned}
$$

with $C=\frac{2 M R}{R-\mu}$ and the last term in the above inequality tending to zero (as $n \rightarrow \infty$ ) for any $\mu<R^{l+1}$, which proves (i).

(ii) By reasoning as in the proof of Theorem 3.6, (iii) (but see also [16], or the book [1] at p.9) we get that the choices $g(q)=(q-\rho)^{-1}$ and $q=\rho^{l+1}$ again gives that $\lim _{n \rightarrow \infty}\left[L_{n-1}(g)(q)-S_{n-1, l}(g)(q)\right] \neq 0$.

(iii) The proof is by reduction to absurdum, that is we suppose that there would exist $l+1$ distinct points $q_{k}$ with $\left\|q_{k}\right\|>\rho^{l+1}, k=1, \ldots, l+1$, such that any three of them do not belong to the same sphere and

$$
\left\|L_{n-1}(f)\left(q_{k}\right)-S_{n-1, l}(f)\left(q_{k}\right)\right\| \leq M, \quad \text { for all } k=1, \ldots, l+1 \text { and } n \geq 1 .
$$

Since $f$ is s-regular in $B(0 ; \rho)$ with a singularity on $\|q\|=\rho$, by [17], p.115, we get

$$
\limsup _{n \rightarrow \infty}\left\|a_{n}\right\|^{1 / n}=\frac{1}{\rho} .
$$

We now have a part of the proof which follows exactly the lines in the proof of Theorem 1 in [2] with some suitable adaptations. We repeat this part of the computation for the reader's convenience. For any $\varepsilon>0$ such that $\rho-\varepsilon>1$ and $(\rho-\varepsilon)^{l+2}>\rho^{l+1}$ there exists $N \in \mathbb{N}, N$ depending on $\varepsilon$, such that $\left\|a_{n}\right\| \leq 1 /(\rho-\varepsilon)^{n}$, for all $n \geq N$. By our hypothesis, $\left\|q_{k}\right\|>\rho^{l+1}, k=1, \ldots, l+1$ and so we can set $\sigma_{1}=\min _{k=1}^{l+1}\left\|q_{k}\right\|$ and $\sigma_{2}=\min _{k=1}^{l+1}\left\|q_{k}\right\|$ where

$$
\rho^{l+1}<\sigma_{1} \leq \sigma_{2}<\rho^{m+1},
$$

where $m$ be the least integer for which the inequality holds. From point (ii) and (15), there exists $M_{1}$ such that

$$
\left\|L_{n-1}(f)\left(q_{k}\right)-\sum_{j=0}^{m-1} P_{n-1, j}(f)\left(q_{k}\right)\right\| \leq M_{1}, \quad n \geq 1, k=1, \ldots, l+1
$$

which, by (13), implies

$$
\left\|\sum_{j=0}^{m-1} P_{n-1, j}(f)\left(q_{k}\right)\right\| \leq M_{2}, \quad n \geq 1, k=1, \ldots, l+1 .
$$


Using $\left\|a_{n}\right\| \leq 1 /(\rho-\varepsilon)^{n}$, we get

$$
\left\|P_{n-1, j}(f)(q)\right\| \leq \frac{n\|q\|^{n}}{(\rho-\varepsilon)^{(j+1) n}}, \quad \forall n \geq N,\|q\|>\rho, j \geq 1 .
$$

Thus, for $l+1 \leq m-1$, we have

$$
\left\|\sum_{j=l+1}^{m-1} P_{n-1, j}(f)(q)\right\| \leq \frac{(m-l-1) n\|q\|^{n}}{(\rho-\varepsilon)^{(l+2) n}}, \quad \forall n \geq N,\|q\|>\rho .
$$

From this inequality and (16), we get

$$
\left\|P_{n-1, j}(f)\left(q_{k}\right)\right\| \leq M_{2}+\frac{(m-l-1) n\left\|q_{k}\right\|^{n}}{(\rho-\varepsilon)^{(l+2) n}}, \quad \forall n \geq N, k=1, \ldots, l+1 .
$$

Note that, for any polynomial $p(q)$ we have $q * p(q)=q p(q)$ and $(q * p(q))_{\mid q=q_{k}}=q_{k} p\left(q_{k}\right)$ and so from the previous inequality it follows that

$$
\left\|q_{k}^{l} P_{n, l}\left(q_{k}\right)-P_{n-1, l}\left(q_{k}\right)\right\| \leq M_{3}+\frac{M_{4} n\left\|q_{k}\right\|^{n}}{(\rho-\varepsilon)^{(l+2) n}}, \quad \forall n \geq N, k=1, \ldots, l+1,
$$

and that

$$
q^{l} P_{n, l}(f)(q)-P_{n-1, l}(f)(q)=\sum_{j=n}^{l+n} q^{j} a_{l n+j}-\sum_{j=0}^{l-1} q^{j} a_{l n+j} .
$$

The last summand in (18) is bounded at the points $q_{k}$, while the first summand evaluated at $q_{k}$ satisfies

$$
\left\|\sum_{j=n}^{l+n} q_{k}^{j} a_{l n+j}\right\| \leq M_{5}+\frac{M_{4} n\left\|q_{k}\right\|^{n}}{(\rho-\varepsilon)^{(l+2) n}} .
$$

Dividing by $\left\|q_{k}\right\|^{n}$ and using the definition of $\sigma_{1}$ we have

$$
\left\|\sum_{j=0}^{l} q_{k}^{j} a_{(l+1) n+j}\right\| \leq \frac{M_{5}}{\sigma_{1}^{n}}+\frac{M_{4} n}{(\rho-\varepsilon)^{(l+2) n}}, \quad \forall n \geq N, k=1, \ldots, l+1 .
$$

Now set $\left.\tau:=\max \left\{1 / \sigma_{1} ; 1 /(\rho-\varepsilon)^{l+2}\right)\right\}$. Then by (15) we have $\tau<1 / \rho^{l+1}$. We now consider the system of $l+1$ linear equations where the unknowns are the coefficients $a_{(l+1) n+j} \in \mathbb{H}$, i.e. we consider

$$
\sum_{j=0}^{l} q_{k}^{j} a_{(l+1) n+j}:=w_{k, n}, \quad k=1,2, \ldots, l+1,
$$

where

$$
\left\|w_{k, n}\right\| \leq M_{6} n \tau^{n}, \quad \forall n \geq N, k=1, \ldots, l+1 .
$$

Consequently, we can write the system in the form (6) whose matrix of coefficients is a Vandermonde matrix of the distinct quaternions $q_{k}, k=1, \ldots, l+1$, such that any three of them do not belong to the same sphere. Therefore, according to Theorem 2.10, the above 
system has a unique solution for $a_{(l+1) n+j}, j=0, \ldots, l$, given by an analogous of the Cramer's method, see [8, Theorem 2.3]. Thus, each $a_{(l+1) n+j}$ is a linear combination (with coefficients depending only on $\left.q_{k}, k=1, \ldots, l+1\right)$ of $w_{k, n}$.

Taking into account (19), this immediately implies that

$$
\left\|a_{(l+1) n+j}\right\| \leq M_{7} n \tau^{n}, \quad n \geq N, k=1, \ldots, l+1 .
$$

This last inequality implies that $\lim \sup \left\|a_{n}\right\|^{1 / n} \leq \tau^{1 /(l+1)}<\frac{1}{\rho}$, which contradicts (14).

Remark 3.8 Note that for $l=1$ we recapture Theorem 3.6.

In the case of complex variable, a converse of the Walsh's result was obtained in [18]. The converse result in the quaternionic setting can be stated as follows.

THEOREM 3.9 Let $\rho>1$, l a positive integer, $f: \overline{B(0 ; 1)} \rightarrow \mathbb{H}$ be an s-regular function on $B(0 ; 1)$, i.e. $f(q)=\sum_{k=0}^{\infty} q^{k} a_{k}$, for all $q \in B(0 ; 1)$ and continuous in $\overline{B(0 ; 1)}$. If the sequence $\left(L_{n-1}(f)(q)-S_{n-1, l}(f)(q)\right)_{n \in \mathbb{N}}$, considered in the statement of Theorem 3.7 is uniformly bounded in every closed subset of $B\left(0 ; \rho^{l+1}\right)$, then $f$ can be slice regularly prolonged to $B(0 ; \rho)$, i.e $f: B(0 ; \rho) \rightarrow \mathbb{H}$ is an s-regular function on $B(0 ; \rho)$.

Exactly as in the complex case in [18], the proof of this result requires the following two lemmas.

Lemm A 3.10 Let $f: \overline{B(0 ; 1)} \rightarrow \mathbb{H}$ be s-regular on $B(0 ; 1)$, i.e. $f(q)=\sum_{k=0}^{\infty} q^{k} a_{k}$ for all $q \in B(0 ; 1)$, and continuous in $\overline{B(0 ; 1)}$. Then we have

$$
L_{n-1}(f)(q)-S_{n-1, l}(f)(q)=L_{n-1}\left(\sum_{k=l n}^{\infty} q^{k} a_{k}\right)(q), l, n \in \mathbb{N}, q \in B(0 ; 1)
$$

Proof The proof is by induction. Indeed, for $l=1$ we get $S_{n-1,1}(f)(q)=P_{n-1,0}(f)(q)=$ $\sum_{k=0}^{n-1} q^{k} a_{k}$. According to Proposition 2.9 and Theorem 2.10, $L_{n-1}(f)(q)$ is the unique polynomial of degree $\leq n-1$ coinciding with $f$ on the $n$ roots of unity, which immediately implies that $L_{n-1}(P)(q)=P(q)$, for any polynomial $P$ of degree $\leq n-1$ and therefore $S_{n-1,1}(f)(q)=\sum_{k=0}^{n-1} q^{k} a_{k}=L_{n-1}\left(\sum_{k=0}^{n-1} q^{k} a_{k}\right)(q)$. The linearity of $L_{n-1}$ follows from its definition and this immediately proves the case $l=1$.

Now, suppose that the relationship is valid for $l-1$. Since $L_{n-1}(g)(q)$ depends only on the $n$ roots of unity and on the values of $g$ taken on the $n$ roots of unity, it is obvious that $L_{n-1}(g)(q)=L_{n-1}\left(e_{n m} g\right)(q)$, for all $m, n \in \mathbb{N}$ (here $\left.e_{n m}(q)=q^{n m}\right)$. So, again by the linearity of $L_{n-1}$, we have

$$
\begin{aligned}
L_{n-1}(f)(q)-S_{n-1, l}(f)(q) & =L_{n-1}(f)(q)-S_{n-1, l-1}(f)(q)-P_{n-1, l-1}(f)(q) \\
& =L_{n-1}\left(\sum_{k=(l-1) n}^{\infty} q^{k} a_{k}\right)(q)-\sum_{k=0}^{n-1} q^{k} a_{k+(l-1) n}
\end{aligned}
$$




$$
\begin{aligned}
& =L_{n-1}\left(\sum_{k=(l-1) n}^{\infty} q^{k} a_{k}\right)(q)-L_{n-1}\left(\sum_{k=(l-1) n}^{l n-1} q^{k} a_{k}\right)(q) \\
& =L_{n-1}\left(\sum_{k=l n}^{\infty} q^{k} a_{k}\right)(q) .
\end{aligned}
$$

Lемм 3.11 If $a_{k} \in \mathbb{H}, k=0,1, \ldots$, then the following relation hold:

$$
\begin{aligned}
a_{\left(3 \ell^{2}+1\right) m+p}-a_{\left(6 \ell^{2}+1\right) m+p}= & \sum_{j=\ell}^{2 \ell-1} a_{(3 j \ell+1) m+p}+\sum_{s=\ell+1}^{2 \ell-1} \sum_{j=\ell}^{2 \ell-1} a_{(3 j s+2 \ell+s+1) m+p} \\
& -\sum_{s=\ell}^{2 \ell-1} \sum_{j=\ell}^{2 \ell-1} a_{(3 j s+2 \ell+j+1) m+p}
\end{aligned}
$$

for $\ell=2,3, \ldots, p=0,1, \ldots, m=1,2, \ldots$

Proof Since it is only simple manipulation of algebraic sums of the coefficients $a_{k}$, it is identical to the proof in the complex case, see [18].

Proof of theorem 3.9 A very careful examination of the proof in the complex case of Theorem 1 in [18], shows that it is not difficult to see that replacing everywhere the modulus $|\cdot|$ with the norm $\|\cdot\|$, from the above Lemmas 3.10,3.11, the properties of the interpolating polynomials $L_{n-1}$ and the representations under Cauchy integral forms of the coefficients of a s-regular function (including the polynomials) and by following exactly the lines in the proof of Theorem 1 in [18], p.272-276, we arrive at the conclusion that

$$
\lim \sup _{n \rightarrow \infty}\left\|a_{n}\right\|^{1 / n} \leq r^{-1 /\left(3 l^{2}+1\right)} .
$$

This means that there exists $\rho^{\prime}>1$ such that $f$ can be prolonged analytically in $B\left(0 ; \rho^{\prime}\right)$ and has a singularity on $\left\{\|q\|=\rho^{\prime}\right\}$. Then, by Theorem 3.7, (iii), the sequence $\left(L_{n-1}(f)(q)-\right.$ $\left.S_{n-1, l}(f)(q)\right)_{n \in \mathbb{N}}$ can be bounded in at most $l$ points $q_{k}, k=1, \ldots, l$, such that any three of them do not belong to the same sphere and satisfy $\left\|q_{k}\right\|>\rho^{l+1}, k=1, \ldots, l$. Comparing this with the hypothesis of Theorem 3.9, it necessarily implies that $\rho \leq \rho^{\prime}$, that is $f$ is s-regular on $B(0 ; \rho)$.

\section{References}

[1] Jakimovski A, Sharma A, Szabados J. Walsh equiconvergence of complex interpolation polynomials. New York: Springer; 2006.

[2] Saff EB, Varga RS. A note on the sharpness of J.L. Walsh's theorem and its extensions for interpolation in the roots of unity. Acta Math. Hung. 1983;41:371-377.

[3] Niven I. Equations in quaternions. Am. Math. Monthly. 1941;48:654-661.

[4] Lam TY. A first course in noncommutative rings. New York: Springer-Verlag; 1991.

[5] Walsh JL. Interpolation and approximation by rational functions in the complex domain. Vol. XX. 5th ed. Providence (RI): American Matematical Society Colloquium Publications; 1969. 
[6] Colombo F, Sabadini I, Struppa DC. Noncommutative functional calculus. Theory and applications of slice hyperholomorphic functions. Basel: Birkhäuser; 2011.

[7] Hou RM, Zhao XQ, Wang LT. The double determinant of Vandermonde's type over quaternion field. Appl. Math. Mech. (English Ed.). 1999;20:1046-1053.

[8] Chen L. Definition of determinant and Cramer solution over quaternion field. Acta Math. Sinica New Series. 1991;7:171-180.

[9] Gentili G, Struppa DC. A new theory of regular functions of a quaternion variable. Adv. Math. 2007;216:279-301.

[10] Gordon B, Motzkin TS. On the zeros of polynomials over division rings. Trans. Am. Math. Soc. 1965;116:218-226.

[11] Beck B. Sur les équations polynomiales dans les quaternions. Enseign. Math. 1979;25:193-201.

[12] Lam TY. A general theory of Vandermonde matrices. Exposition. Math. 1986;4:193-215.

[13] Lam TY, Leroy A. Vandermonde and Wronskian matrices over division rings. J. Algebra. 1988;119:308-336.

[14] Opfer G. Polynomials and Vandermonde matrices over the field of quaternions. Elect. Trans. Num. Anal. 2009;36:9-16.

[15] Alpay D, Colombo F, Sabadini I. Pontryagin de Branges Rovnyak spaces of slice hyperholomorphic functions. J. Anal. Math. 2013;121:87-125.

[16] Cavaretta AS, Sharma A, Varga RS. Interpolation on the roots of unity: an extension of a theorem of J.L. Walsh. Results Math. 1980;3:155-191.

[17] Gentili G, Stoppato C. Power series and analyticity over the quaternions. Math. Ann. 2012;352:113-131.

[18] Szabados J. Converse results in the theory of overconvergence of complex interpolating polynomials. Analysis. 1982;2:267-280. 